\title{
APPENDIX FIVE
}

\section{INDIVIDUALS ACCUSED OF \\ WITCHCRAFT, 1543-46}

\begin{tabular}{|c|c|c|c|}
\hline Name & $\operatorname{Alias}(e s)$ & Personnel details & Trial result \\
\hline Claude Vulliemoz & Grangiez & & \\
\hline Katherine & & $\begin{array}{l}\text { Wife of Antoine du Fossal, from } \\
\text { Gex }\end{array}$ & $\begin{array}{l}\text { Beaten, } \\
\text { rebanished }\end{array}$ \\
\hline Rolette & & $\begin{array}{l}\text { Widow of Pierre Pilliciez, from } \\
\text { Baleyson }\end{array}$ & Rebanished \\
\hline Pernette Dorsier & & $\begin{array}{l}\text { Daughter of Monet, widow of } \\
\text { François Dunant }\end{array}$ & Banished \\
\hline Louis Verchiere & & From Satigny & \\
\hline Andréaz & & Widow of Pierre Juget, from Peney & \\
\hline Morys & Mauris & Wife of Borgeaulx, from Satigny & Executed \\
\hline Aymed Darvex & & $\begin{array}{l}\text { Confessed to being a witch for six } \\
\text { years and and having renounced } \\
\text { God and paid homage to Satan, } \\
\text { legal opinion divided }\end{array}$ & Banished \\
\hline Henry du Gerdil & & From Peney & Fined, released \\
\hline Claude Malliez & & From Satigny & \\
\hline Lyonardaz & & $\begin{array}{l}\text { Widow of Claude Donne (from } \\
\text { Bourdigny), from Peney }\end{array}$ & $\begin{array}{l}\text { Banished, beaten, } \\
\text { rebanished }\end{array}$ \\
\hline Tevenaz Paris & & From Peney & Banished \\
\hline Unnamed woman & & Mother of Henry Juget & Died in custody \\
\hline Bastien de Nantua & Le Bon Herige & Barber-surgeon & Banished \\
\hline Charles de Soye & & Barber-surgeon, diviner & Freed \\
\hline Unnamed woman & & $\begin{array}{l}\text { Widow of Jean Pasteur of } \\
\text { Vandoeuvres, amulet use }\end{array}$ & \\
\hline
\end{tabular}

